\title{
CENIZAS DE BIOMASA: UNA OPORTUNIDAD PARA INCREMENTAR LOS BENEFICIOS MEDIOAMBIENTALES Y TECNOLÓGICOS DE HORMIGONES CON CEMENTO PORTLAND Y GEOPOLIMÉRICOS
}

\author{
J. ROSELLÓ ${ }^{1}$, M. V. BORRACHERO ${ }^{2}$, J. MONZÓ², A. MELLADO ${ }^{2}$, \\ M. P. SANTAMARINA ${ }^{1}$, L. SORIANO ${ }^{2}$, A. FONT ${ }^{2}$, J. PAYÁ ${ }^{1}$ \\ ${ }^{1}$ Departamento de Ecosistemas Agroforestales, Universitat Politècnica de València (España) \\ ${ }^{2}$ Instituto de Ciencia y Tecnología del Hormigón (ICITECH), Universitat Politècnica de Valéncia (España)
}

\section{RESUMEN}

El uso de las cenizas de biomasa es un recurso potencial para la elaboración de materiales cementantes. La composición química de dichas cenizas influye en sus posibles aplicaciones. En este trabajo se comparan las características de varias cenizas procedentes de las siguientes biomasas: hoja de bambú, paja de caña de azúcar, hueso de oliva y cáscara de almendra. Por una parte, se demuestra la potencialidad puzolánica de las cenizas con elevados contenidos en sílice; por otra parte, se analiza la potencialidad de las cenizas de carácter alcalino, ricas en óxidos de potasio y de calcio. Finalmente se hace una evaluación de los aspectos medioambientales y de durabilidad en función de su dosificación y su composición química.

Palabras clave: activación alcalina; reacción puzolánica; fitolitos; espodograma; huella de carbono.

\begin{abstract}
The use of biomass ashes is a potential resource for the production of cementing materials. The chemical composition of these ashes influences their possible applications. In this work, the characteristics of several ashes coming from the following biomasses are compared: bamboo leaf, sugar cane straw, olive stone and almond shell. On the one hand, the pozzolanic potential of the ashes with high silica content is demonstrated; on the other hand, the potentiality of alkaline ashes, rich in potassium and calcium oxides, is analyzed. Finally, an evaluation of the environmental and durability aspects according to their dosage and chemical composition is made.
\end{abstract}

Keywords: alkaline activation; pozzolanic reaction; phytoliths; spodogram; carbon footprint.

\section{RESUMO}

O uso de cinzas de biomassa é um recurso potencial para a produção de materiais cimentícios. A composição química dessas cinzas influencia suas possíveis aplicações. Neste trabalho, são comparadas as características de várias cinzas provenientes das seguintes biomassas: folha de bambu, palha de cana-de-açúcar, caroço de azeitona e casca de amêndoa. Por um lado, o potencial pozolânico das cinzas com alto teor de sílica é demonstrado; por outro lado, analisa-se a potencialidade das cinzas alcalinas, ricas em óxidos de potássio e cálcio. Finalmente, é feita uma avaliação dos aspectos ambientais e de durabilidade de acordo com sua dosagem e composição química.

Palavras-chave: ativação alcalina; reação pozolânica; fitólitos; espodograma; pegada de carbono. 


\section{INTRODUCCIÓN}

El creciente interés por la disminución de las emisiones de $\mathrm{CO}_{2}$ asociadas a la fabricación de cementos (Andrew, 2018) ha provocado el desarrollo de diversas líneas tecnológicas: i) modificaciones del proceso de clinkerización; ii) diseño nuevos cementos basados en otro tipo de clinker (belítico y de sulfoaluminato de calcio); iii) desarrollo de cementos binarios y ternarios con adiciones minerales; iv) diseño de cementos de activación alcalina (CAA) y geopolímeros (GP). En los dos últimos casos, existe un potencial para el uso de residuos generados en actividades industriales, agrícolas, energéticas y de descontaminación. La sustitución parcial del clinker de cemento Portland (habitualmente entre $10 \mathrm{y}$ $40 \%$ en masa) por materiales suplementarios de carácter puzolánico, hidráulico o inerte puede reducir la huella de carbono de forma significativa cuando dichos materiales son de residuos: tal es el caso de cenizas volantes de carbón, escorias de alto horno o humo de sílice; sin embargo, sería mucho menos ventajoso el uso de materiales como el metacaolín, por ser un componente que requiere gran energía en su síntesis. La preparación de CAA o GP puede ofrecer más ventajas (Mellado et al., 2014) dado que en estos casos se elimina el uso de clinker que es sustituido en su totalidad por residuos.

Entre los residuos con mayor potencial de utilización destacan las cenizas generadas por la combustión de biomasa, debido a que en algunos casos pueden cumplir con el grado de reactividad adecuado. La biomasa está compuesta mayoritariamente por sustancias orgánicas naturales, y pueden proceder de plantas o animales. Las plantas tienen capacidad para absorber ciertos elementos químicos del suelo, e incorporarlos a sus sistemas de tejidos. Entre estos elementos químicos destacan $\mathrm{Ca}, \mathrm{Si}, \mathrm{Al}, \mathrm{Ti}, \mathrm{Fe}, \mathrm{Mg}$, Na, K, S y P (James et al., 2012). La combustión de la biomasa permite la obtención de calor de forma directa o bien la obtención de energía eléctrica o mecánica a través de procesos de conversión. La biomasa se considera un combustible neutro a nivel de emisiones de $\mathrm{CO}_{2}$, puesto que se trata de una materia renovable, aunque algunos autores empiezan a cuestionar esa neutralidad, dado que son necesarios consumos de energía adicionales (Johnson, 2009).

Todo ello despierta un gran interés en el ámbito de la generación de energía debido al proceso de descarbonización: en la actualidad, más del 10\% de la energía generada tiene como origen la biomasa (World Energy Council, 2019).

La transformación de la biomasa por combustión, para la obtención de energía, supone la generación de cantidades significativas de cenizas, aspecto que ya empieza a ser tenido en cuenta como un problema medioambiental y que tiene consecuencias económicas. Si hubiera una explotación completa de la biomasa generada anualmente a nivel mundial, se estima que existe un potencial productivo de 480 millones de toneladas de ceniza (Jankovsky et al., 2017). Las características químicas, físicas y mineralógicas de las cenizas pueden cambiar en función de la naturaleza de la biomasa y de las condiciones de combustión. Así, por ejemplo, Jenkins et al. (1998) encontraron que el contenido de cenizas entre una selección de especies de cultivo variaba entre 1.7\% (madera de sauce) y 20.26\% (cáscara de arroz). Por otra parte, las elevadas temperaturas de calcinación pueden potenciar la cristalización de algunos compuestos; así, Shen et al. (2011) encontraron que calcinaciones de la cáscara de arroz a $700^{\circ} \mathrm{C}$ durante tiempos cortos (menos de 1 hora) producían una sílice amorfa, mientras que incrementos en el tiempo ( 3 horas) o incrementos en la temperatura $\left(800^{\circ} \mathrm{C}, 0.5\right.$ horas) generaban cenizas con importantes contenidos de cristobalita.

En este trabajo se presentan las características de diversas cenizas obtenidas de biomasas de hoja de bambú, paja de caña de azúcar, hueso de oliva y cáscara de almendra, con el objeto de comparar diferentes situaciones y valorar la contribución que pueden tener estas cenizas en el desarrollo de materiales cementantes más sostenibles. 


\section{PROCEDIMIENTO EXPERIMENTAL}

Se seleccionaron diversas cenizas a partir de biomasas. Dichas cenizas se obtuvieron por medio de calcinación en mufla, a temperaturas entre $350^{\circ} \mathrm{C}$ y $850^{\circ} \mathrm{C}$, con el objeto de eliminar la mayor parte de la fracción orgánica de la materia vegetal.

Hojas de bambú (Bambusa vulgaris). Esta caña va perdiendo las hojas más viejas con el tiempo, que se van depositando al pie de la misma. Estas hojas se recogieron de unos ejemplares situados el Ilha Solteira (São Paulo, Brasil). Las hojas se secaron a $105^{\circ} \mathrm{C}$ y luego se calcinaron a $850^{\circ} \mathrm{C}$ durante 1 hora, obteniendo la ceniza correspondiente (CHB, ceniza de hoja de bambú).

Paja de caña de azúcar (Saccharum officinarum). Se recolectó (hojas y vainas) en un campo en las cercanías de Ilha Solteira (São Paulo, Brasil). El material se limpió del polvo del suelo y se secó a $105^{\circ} \mathrm{C}$. Las hojas secas se calcinaron a $350^{\circ} \mathrm{C}$ y $450^{\circ} \mathrm{C}$ en una mufla por 1 hora, obteniendo la ceniza de paja de caña de azúcar (CPCA).

Huesos de oliva (Olea europaea). Se recolectaron huesos de oliva en la empresa Aceites Sahúco (España) que son utilizados en dicha empresa para las calderas de vapor. Se calcinaron estas muestras a $500^{\circ} \mathrm{C}$ durante 3 horas en mufla, obteniendo la ceniza de hueso de oliva (CHO).

Cáscaras de almendra (Prunus dulcis). Se recolectaron estas cáscaras en la empresa Borges (Altura, España), las cuales son utilizadas como combustible para el secado de la almendra. Para obtener las cenizas, se calcinaron las cáscaras a $500^{\circ} \mathrm{C}$ durante 3 horas en mufla, obteniendo la correspondiente ceniza (Ceniza de cáscara de almendra, CCAl).

Las cenizas se analizaron microestructuralmente mediante microscopia óptica y microscopía electrónica de barrido y se ha obtenido su composición química mediante la técnica de análisis SEMEDS.

\section{RESULTADOS}

En los siguientes apartados se muestran los estudios microscópicos y de composición química de las cenizas analizadas.

\subsection{Ceniza de hoja de bambú (CHB).}

En la Figura 1 se muestran dos micrografías de la CHB. Se trata del esqueleto inorgánico remanente después de la calcinación (espodograma), en el que podemos observar diversas estructuras. Destaca unas formas compactas, denominadas fitolitos (Roselló et al, 2015). Estas estructuras consisten en acúmulos de sílice amorfa. Estas acumulaciones se pueden observar sobre el material previo a la calcinación, en un estudio de microscopía óptica. En la Figura 2 (imágenes por microscopía óptica) se muestra que dichos acúmulos no son consecuencia de la eliminación de la materia orgánica y de una coalescencia de la fracción inorgánica, sino que ya se observan previamente a la calcinación. El análisis SEM-EDS sobre uno de estos fitolitos dio una composición de $\mathrm{SiO}_{2}=96.27 \%, \mathrm{~K}_{2} \mathrm{O}=2.07 \%$, $\mathrm{MgO}=0.35 \%, \mathrm{SO}_{3}=0.85 \%$ y Cl$=0.47 \%$. Se identifican dos morfologías de estos fitolitos. En la Figura 1a se muestra la forma "silla de montar" (saddle) y en la Figura 1b se observa la forma "mancuerna" (dumbell). Estos fitolitos están rodeados de una matriz inorgánica formada por los restos de las paredes celulares. La composición química global de la CHB, obtenida por SEM-EDS en muestras calcinadas a $850^{\circ} \mathrm{C}$ muestra claramente la naturaleza ácida de la ceniza: presenta un $87.9 \%$ de $\mathrm{SiO}_{2}$. Los otros componentes de carácter secundario son el $\mathrm{K}_{2} \mathrm{O}(5.4 \%), \mathrm{CaO}(3.2 \%)$ y $\mathrm{Cl}(0.1 \%)$. Por último, trazas de otros elementos $(\mathrm{Mg}, \mathrm{P}, \mathrm{S})$ completan la composición química de la CHB.

El uso de la CHB como material suplementario cementante para la sustitución de cemento Portland en morteros implica la posibilidad de contribución puzolánica debido a la presencia de sílice amorfa (Moraes et al., 2019). 
Así, por ejemplo, en morteros normalizados con sustitución de $25 \%$ por CHB, se obtienen una resistencia mecánica a 28 días que supone el $115 \%$ del motero control. Ello significa que, verdaderamente, la sílice presente es reactiva desde el punto de vista puzolánico. A tiempos cortos (3 días) se alcanza un valor de resistencia a compresión que supone de $90 \%$, a pesar de tener un contenido $25 \%$ menor de cemento y una relación real agua/cemento de 0.67 , frente al 0.5 del control. Ello sugiere que la reactividad puzolánica es muy temprana, por lo que este tipo de ceniza sería útil como alternativa al humo de sílice, de la misma forma que la ceniza molida de cáscara de arroz (Xu et al, 2016).

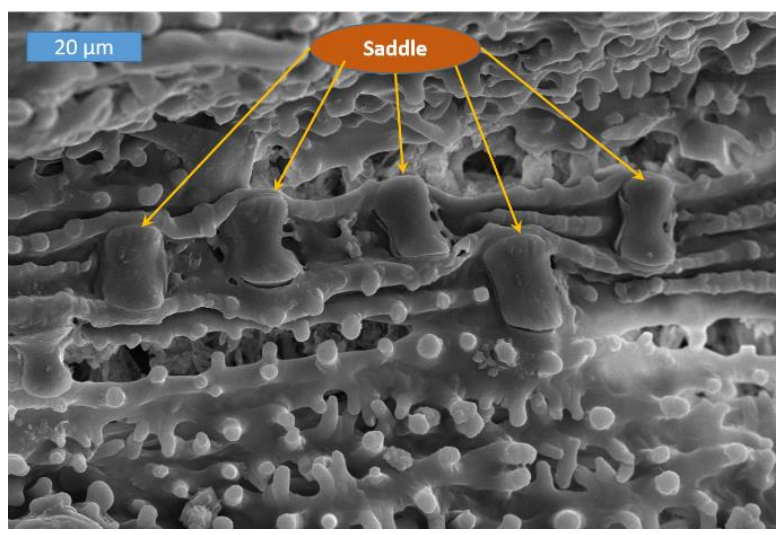

(a)

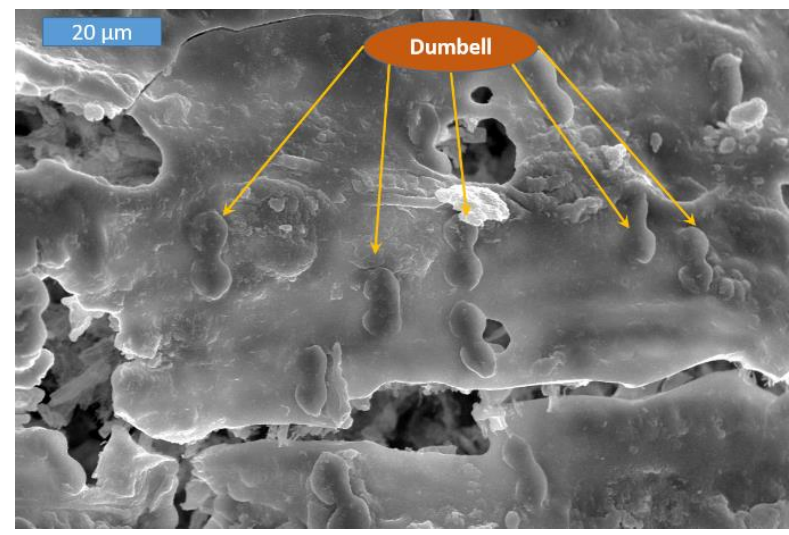

(b)

Figura 1. Micrografías SEM de ceniza de hoja de bambú (CHB) obtenidas a $850^{\circ} \mathrm{C}$ : a) espodograma con fitolitos "saddle"; b) espodograma con fitolitos "dumbell".

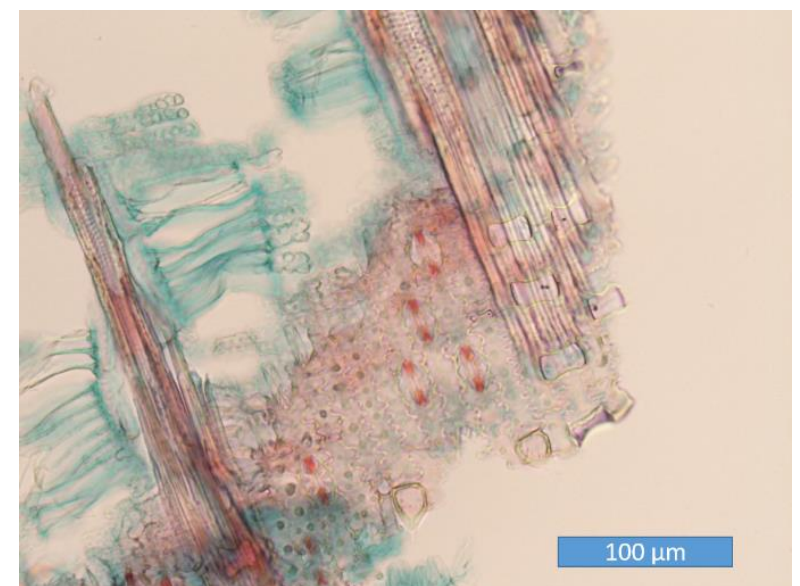

(a)

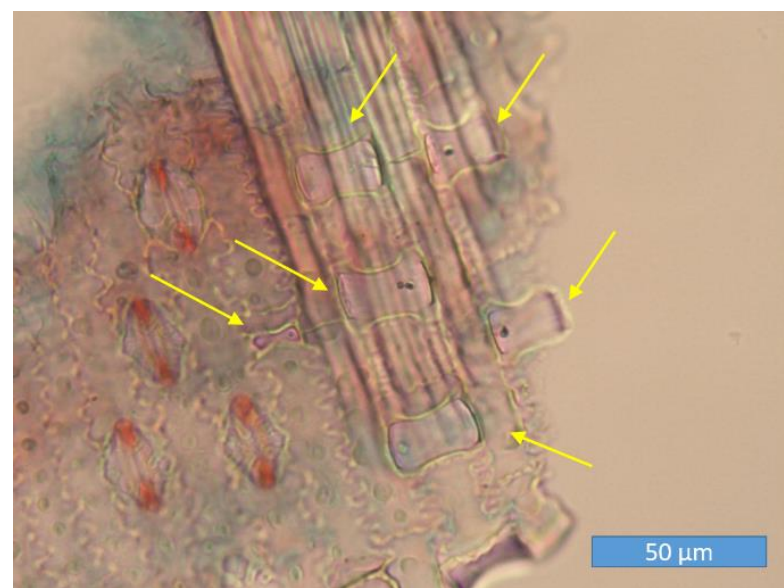

(b)

Figura 2. Sección paradermal de hojas de bambú, antes de la calcinación, observada en microscopía óptica: a) tomada a 200 aumentos; b) tomada a 400 aumentos. Los fitolitos están indicados con flechas.

\subsection{Ceniza de paja de caña de azúcar (CPCA).}

En la Figura 3 se muestran dos micrografías de la CPCA. En la Figura 3a podemos observar que, las cenizas obtenidas a $450^{\circ} \mathrm{C}$, tienen un esqueleto más desordenado que en la $\mathrm{CHB}$. Para mantener el espodograma un poco más intacto, se calcinó la hoja a $350^{\circ} \mathrm{C}$, de manera que se observa (Figura $3 \mathrm{~b}$ ) la disposición ordenada de fitolitos. En este caso, estas estructuras presentan la forma "dumbell". En la Figura 4 se pueden observar los fitolitos en las muestras antes de calcinar. La composición química de los fitolitos también presenta elevados porcentajes de $\mathrm{SiO}_{2}(89.95 \%)$, aunque en este caso la proporción de $\mathrm{K}_{2} \mathrm{O}$ es significativamente mayor que en el caso de $\mathrm{CHB}(9.42 \%)$. Otros elementos que forman parte 
del fitolito son $\mathrm{MgO}(0.25 \%)$ y $\mathrm{Cl}(0.39 \%)$. La composición química media determinada por SEM-EDS muestra claramente que la ceniza no es tan ácida ya que el porcentaje de $\mathrm{SiO}_{2}$ se queda en $32.3 \%$. Se trata por tanto de una ceniza de baja acidez. Los otros componentes que forman parte de la CPCA son: $\mathrm{K}_{2} \mathrm{O}=30.0 \%, \mathrm{Cl}=4.3 \%, \mathrm{CaO}=13.0 \%, \mathrm{MgO}=9.4 \%, \mathrm{P}_{2} \mathrm{O}_{5}=4.2$ y SO $3=6.3 \%$. Debido al contenido en sílice, este material también presenta una reactividad puzolánica interesante (Moraes et al., 2016). La resistencia mecánica de morteros sustituidos con $20 \%$ de cemento Portland es el $95 \%$ del control a 28 días. Sin embargo, a 7 días de curado, dicha resistencia solamente alcanza el $75 \%$ del mortero con solo cemento. Estos datos demuestran que es una puzolana activa, aunque requiere períodos de curado largos para que contribuya al desarrollo de resistencia.

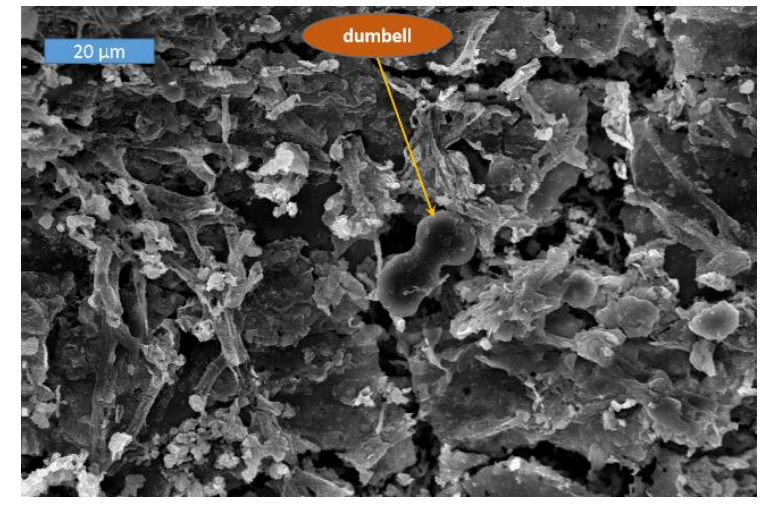

(a)

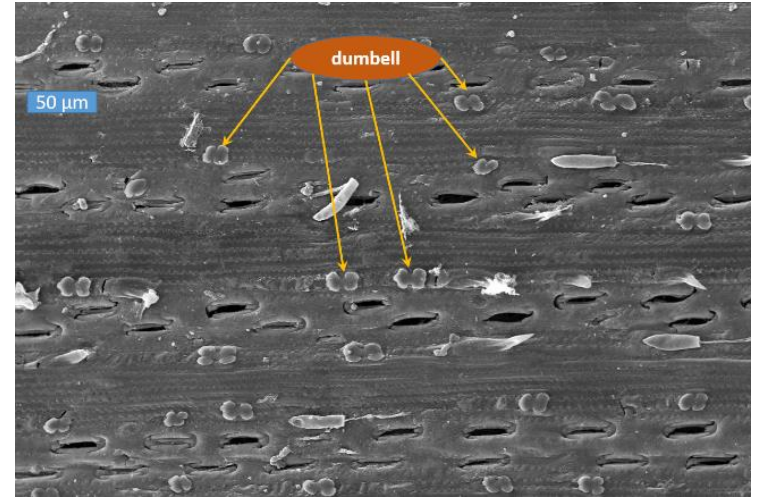

(b)

Figura 3. Micrografías SEM de ceniza de paja de caña de azúcar (CPCA) obtenidas a la temperatura de: a) $450^{\circ} \mathrm{C}$; b) $350^{\circ} \mathrm{C}$.

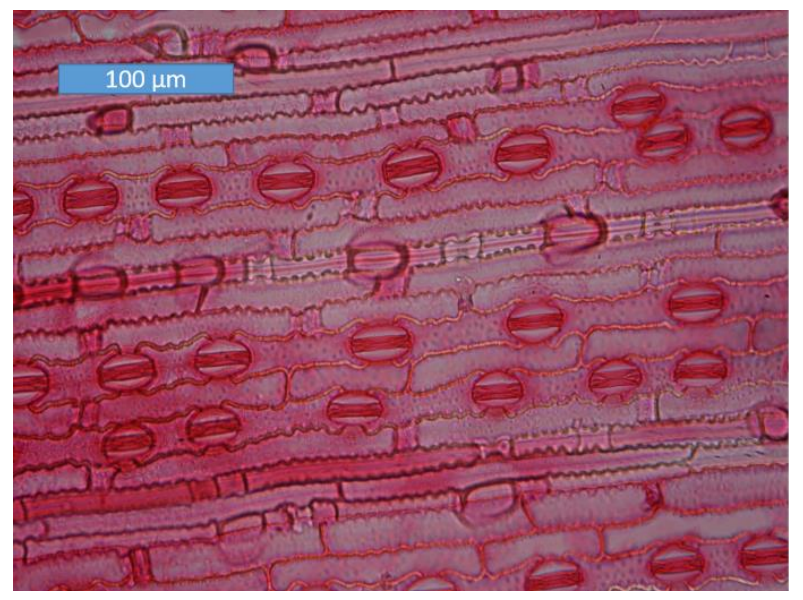

(a)

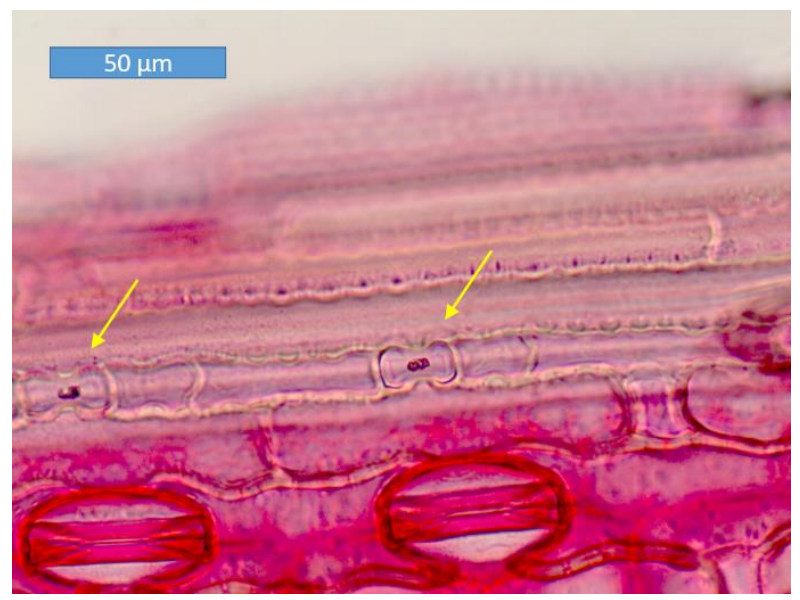

(b)

Figura 4. Sección paradermal de hojas de caña de azúcar, antes de la calcinación, observada en microscopía óptica: a) tomada a 200 aumentos; b) tomada a 400 aumentos. Los fitolitos están indicados con flechas.

\subsection{Ceniza de hueso de oliva (CHO).}

La obtención del aceite de oliva se lleva a cabo por prensado de olivas, en el cual se tritura la pulpa junto al hueso. El hueso de oliva se corresponde con el endocarpo (parte interna del fruto) y después de que se elimine la pulpa residual (exocarpo y mesocarpo), queda en pequeños trozos de varios milímetros (Figura 5a). En la Figura 5b se muestran la parte interior (más lisa) y exterior (más rugosa) del hueso y en las Figuras $5 \mathrm{c}$ y $5 \mathrm{~d}$ se muestra la microestructura bajo el microscopio electrónico. Se puede observar que se trata de un material compacto, de origen esclerenquimático. 
En la Figura 6 se muestran micrografías de las cenizas obtenidas por calcinación del hueso de oliva a $500^{\circ} \mathrm{C}(\mathrm{CHO})$. Se puede observar claramente que se trata de un material con un tamaño de partícula muy reducido: la mayor parte presentan un diámetro inferior a las 20 micras. Casi todas las partículas son de morfología irregular y bastante porosas. Todo ello indica que después de la calcinación, el esqueleto inorgánico queda muy reducido, de modo que se pierde la identidad en la distribución de la fracción inorgánica. En algunos casos se identifica alguna partícula compacta y de caras regulares: dichas partículas están constituidas fundamentalmente por cloruro potásico $(\mathrm{KCl})$, que tiende a formar cristales. La composición química de $\mathrm{CHO}$, determinada por SEM-EDS, demuestra que se trata de cenizas de carácter alcalino, puesto que el contenido en silicio es despreciable $\left(\mathrm{SiO}_{2}=2 \%\right)$. Sin embargo, los contenidos en potasio y calcio son muy elevados: $\mathrm{K}_{2} \mathrm{O}=62.7 \%$ y $\mathrm{CaO}=14.7 \%$. Los otros elementos significativos son $\mathrm{Na}, \mathrm{Mg}, \mathrm{P}, \mathrm{S}$ y $\mathrm{Cl}$ (para este último componente, $0.32 \%$ ).

Este tipo de ceniza no puede actuar como puzolana. Sin embargo, por su potencial alcalino, es muy interesante como sustancia activadora en cementos de activación alcalina. CHO puede sustituir al hidróxido de potasio y al hidróxido de sodio en la activación de escoria de alto horno (Pinheiro et al., 2018). Morteros de escoria de alto horno activados con adición de $25 \%$ de $\mathrm{CHO}$ (7 días de curado, $65^{\circ} \mathrm{C}$ ) presentan una resistencia mecánica (38MPa) que es $150 \%$ la del mortero activado con $\mathrm{KOH} 8 \mathrm{M}$ y $120 \%$ la del activado con $\mathrm{NaOH} 12 \mathrm{M}$ (ambos con relación agua/binder 0.4).

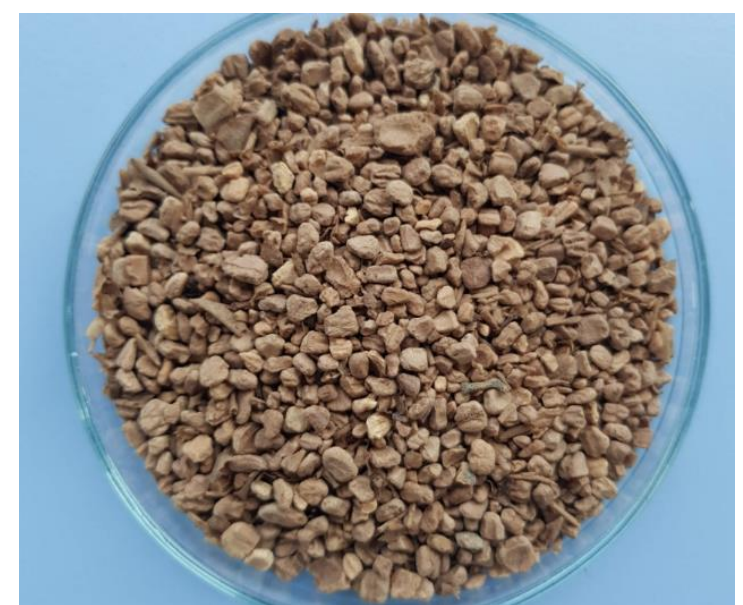

(a)

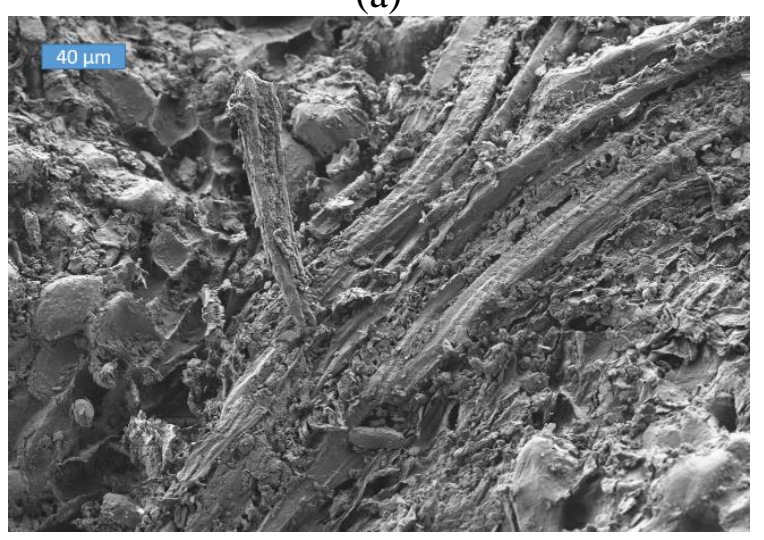

(e)

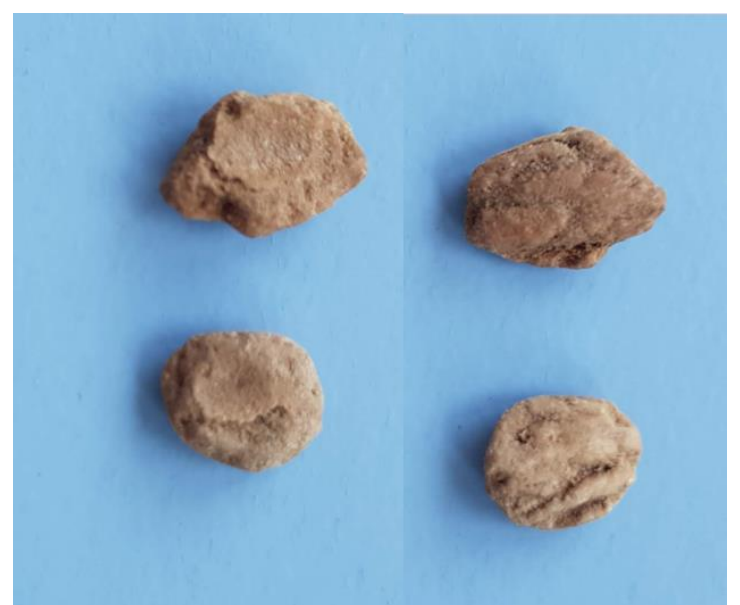

(b)

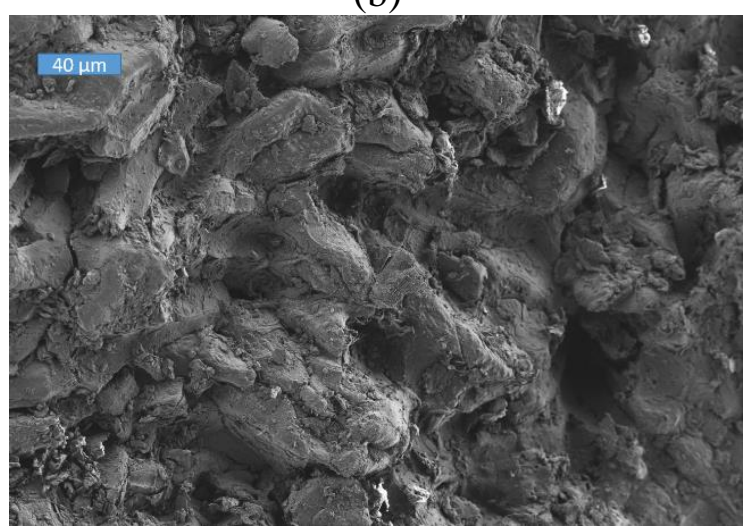

(f)

Figura 5. Imágenes tomadas con cámara digital y microscopio electrónico del hueso de oliva: a) vista general de los trozos de hueso; b) parte interna (izquierda) y parte externa del hueso; c) vista SEM de la parte externa (x300); d) vista SEM de la parte interna (x300). 


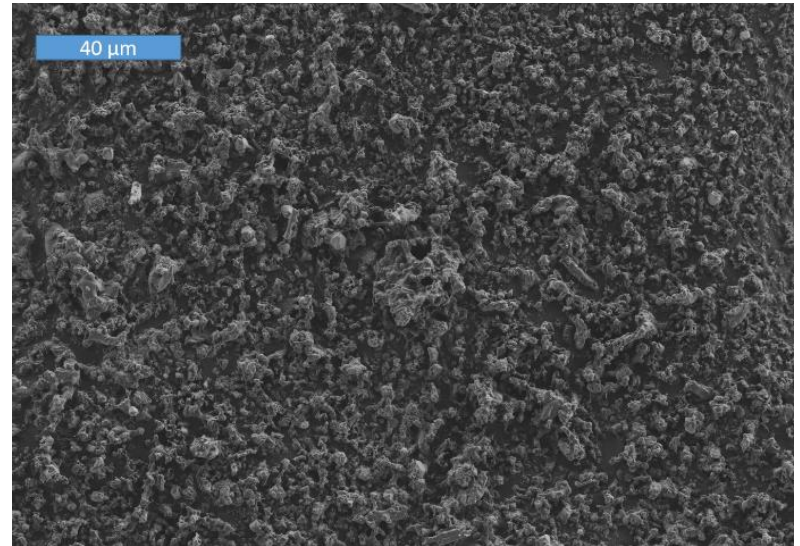

(a)

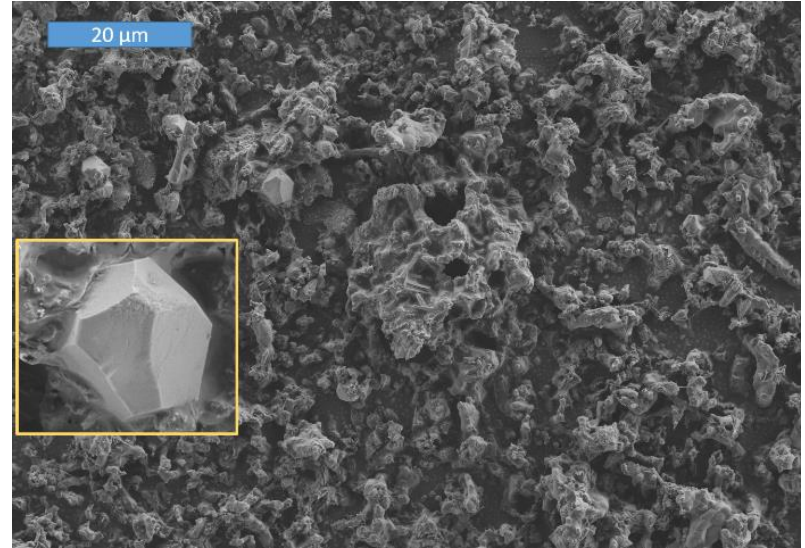

(b)

Figura 6. Micrografías SEM de ceniza de hueso de oliva (CHO) obtenidas a la temperatura de $500^{\circ} \mathrm{C}$ durante 3 horas: a) a 500 aumentos; b) a 1000 aumentos (en el recuadro aparece un cristal de cloruro potásico, $\mathrm{KCl})$.

\subsection{Ceniza de cáscara de almendra (CCAl).}

El fruto del almendro está constituido por una parte exterior carnosa (colfa: exocarpo más mesocarpo) la cual se va secando a medida que madura el fruto y, finalmente, se despega de la cáscara leñosa. La cáscara de almendra es el endocarpo del fruto y rodea a la semilla. El endocarpo, duro y leñoso, está compuesto por tejido esclerenquimático (células pétreas o esclereidas), células de paredes engrosadas, lignificadas y duras. En la Figura 7 se muestran diferentes imágenes de la cáscara.

En la Figura 8 se muestran dos micrografías de la ceniza de cáscara de almendra (CCAl.) también obtenida por calcinación a $500^{\circ} \mathrm{C}$. Se puede observar que la muestra contiene aglomerados de micropartículas, formando estructuras muy porosas. Esos aglomerados están compuestos por mezclas de micropartículas porosas y compactas: estas últimas contiene casi exclusivamente $\mathrm{K}_{2} \mathrm{O}$. La composición química por SEM-EDS muestra que se trata de cenizas alcalinas, con elevados contenidos en potasio y calcio: $\mathrm{K}_{2} \mathrm{O}=40 \%$ y $\mathrm{CaO}=43 \%$. Apenas presenta $2 \%$ de $\mathrm{SiO}_{2}$ y solamente $0.29 \%$ de $\mathrm{Cl}$. $\mathrm{El}$ otro componente significativo es el magnesio $(\mathrm{MgO}=8 \%)$.

Esta ceniza es candidata a poder activar escoria de alto horno, debido a su elevada alcalinidad. Morteros de escoria de alto horno activados con adición de $20 \%$ de CCAl ( 7 días de curado, $65^{\circ} \mathrm{C}$ ) presentan una resistencia mecánica (37MPa) que es $145 \%$ respecto a la de un mortero activado con KOH 8M.

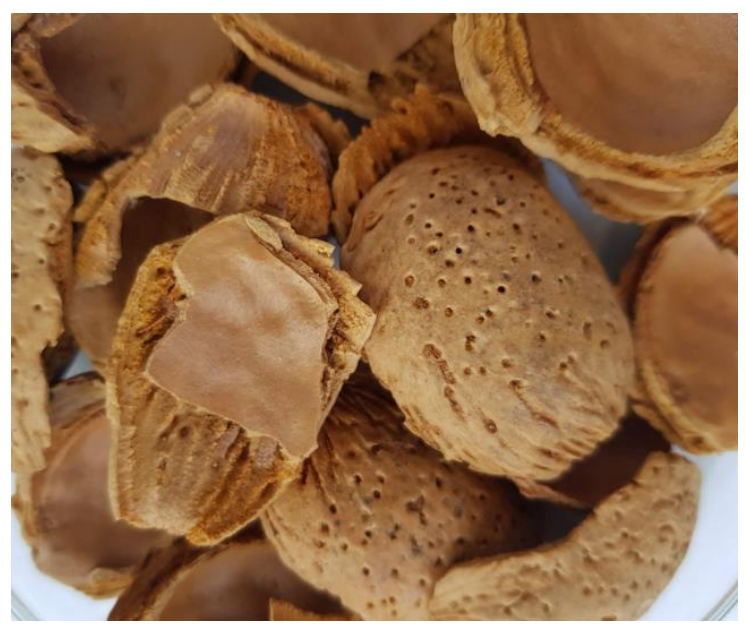

(a)

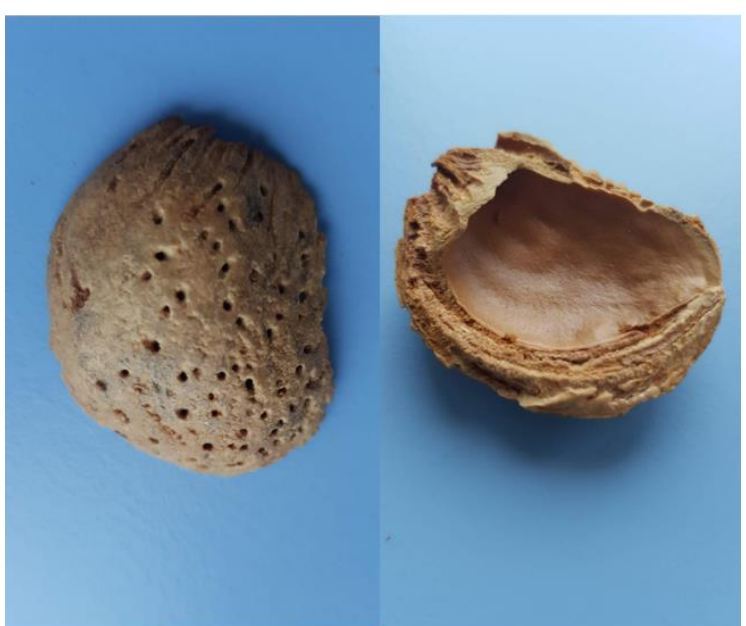

(b) 


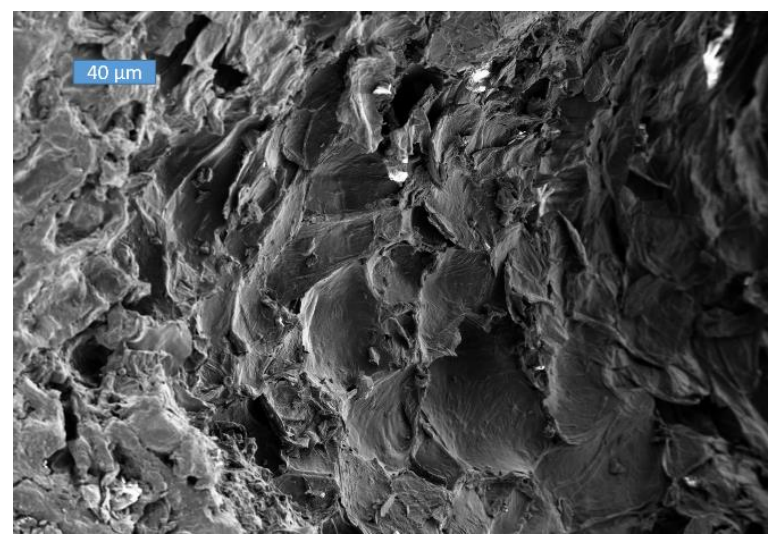

(e)

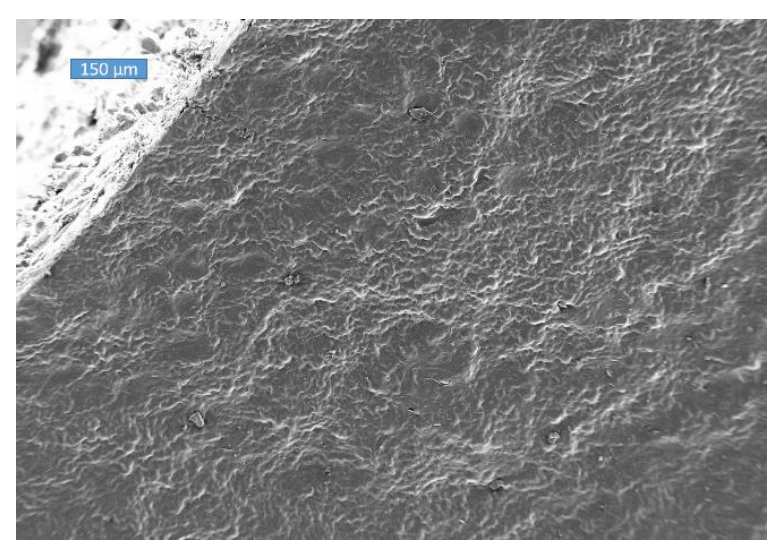

(f)

Figura 7. Imágenes tomadas con cámara digital y microscopio electrónico de la cáscara de almendra: a) vista general de trozos de la cáscara; b) cara externa (izquierda) y cara interna de la cáscara; c) vista SEM de la parte externa (x300); d) vista SEM de la parte interna (x75).

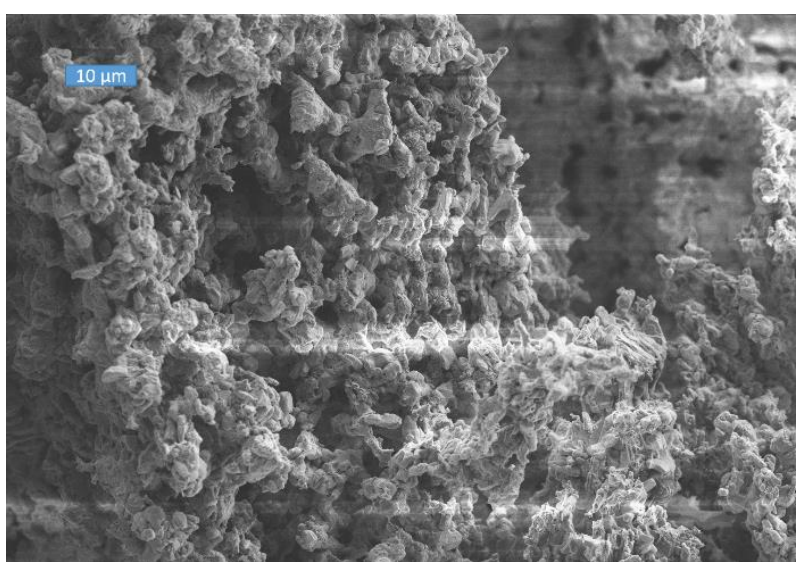

(a)

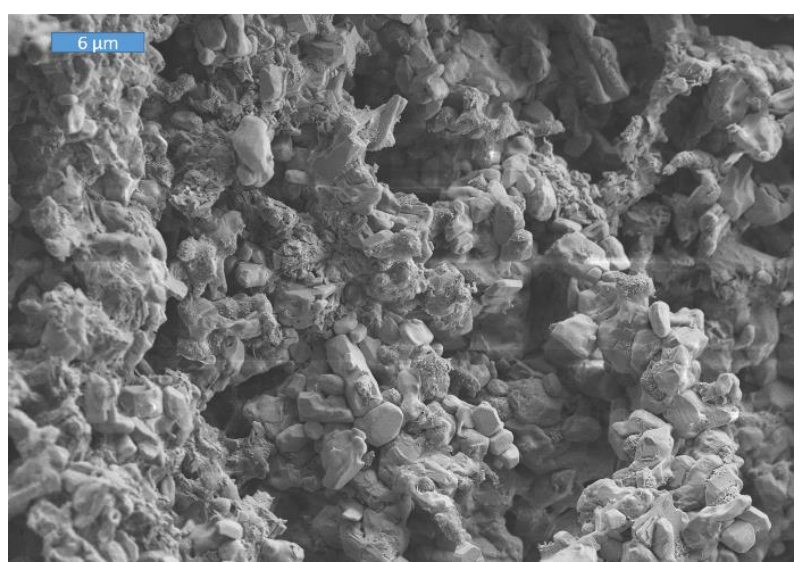

(b)

Figura 8. Micrografías SEM de ceniza de cáscara de almendra (CCAl) obtenidas a la temperatura de $500^{\circ} \mathrm{C}$ durante 3 horas: a) a 1000 aumentos; b) a 2000 aumentos.

\section{DISCUSIÓN}

La naturaleza química de las cenizas depende en gran medida de las características y origen de la biomasa vegetal. Por otra parte, teniendo en cuenta que, en una primera aproximación, las cenizas de biomasa vegetal no llevan asociada ninguna cantidad de $\mathrm{CO}_{2}$ emitido, es posible diseñar conglomerantes para la construcción que posean una huella de carbono baja. Finalmente, la presencia de algunos componentes químicos puede activar los procesos de degradación del hormigón o de la armadura embebida. Todos estos aspectos se discuten a continuación.

\subsection{Composición química de las cenizas de biomasa vegetal}

Como se ha podido comprobar, es posible encontrar cenizas de elevado contenido en sílice (como el caso de $\mathrm{CHB}$ ) y cenizas con alto contenido en potasio y calcio (como los casos de $\mathrm{CHO}$ y $\mathrm{CCAl}$ ). Vassilev et al. (2012) han definido un diagrama ternario para ubicar los distintos tipos de cenizas procedentes de biomasa y de combustibles fósiles. En la Figura 9 se muestra un esquema del diagrama ternario. Dicho diagrama tiene definidos tres vértices, que representan los contenidos de diferentes sustancias (en forma de óxidos) que componen la ceniza. En uno de los vértices $(\sigma)$ se agrupan los 
óxidos $\mathrm{SiO}_{2}, \mathrm{Al}_{2} \mathrm{O}_{3}, \mathrm{Fe}_{2} \mathrm{O}_{3}, \mathrm{Na}_{2} \mathrm{O}$ y $\mathrm{TiO}_{2}$. Las cenizas de hoja de bambú se encontrarían cerca de este vértice, puesto que son muy ricas en $\mathrm{SiO}_{2}$, y su posición estaría dentro de lo que se denomina zona $\mathrm{S}$. En los otros dos vértices aparecen otros óxidos: por un lado, la suma de $\mathrm{CaO}, \mathrm{MgO}$ y $\mathrm{MnO}$ (vértice $\tau$ ) y por otro lado la suma de $\mathrm{K}_{2} \mathrm{O}, \mathrm{P}_{2} \mathrm{O}_{5}, \mathrm{SO}_{3}$ y $\mathrm{Cl}_{2} \mathrm{O}$ (vértice $\kappa$ ).

$\mathrm{CHO}$ y CCAl se situarían en dicho diagrama en el lado que une los vértices $\tau$ y $\kappa$, puesto que $\mathrm{CaO}$ y $\mathrm{K}_{2} \mathrm{O}$ son los óxidos mayoritarios. Específicamente, $\mathrm{CHO}$ estaría en la zona $\mathrm{K}$ y CCAl en la zona CK. Finalmente, la CPCA se situaría también en la zona K; sin embargo, es evidente que su comportamiento es más de tipo puzolánico, por lo que se propone, a la vista de las aplicaciones de las cenizas de biomasa en materiales cementantes, una alteración del diagrama ternario original, de acuerdo con la Figura 10. Se ha diferenciado la zona $\mathrm{K}$ en otras dos, una de mayor alcalinidad (nueva zona K) y otra de baja acidez (Zona K-BA). Se ha situado una línea de separación para cenizas que contengan un porcentaje menor de $10 \%$ para los componentes $\sigma$, de modo que la zona inferior ha quedado diferenciada según la Figura 10.

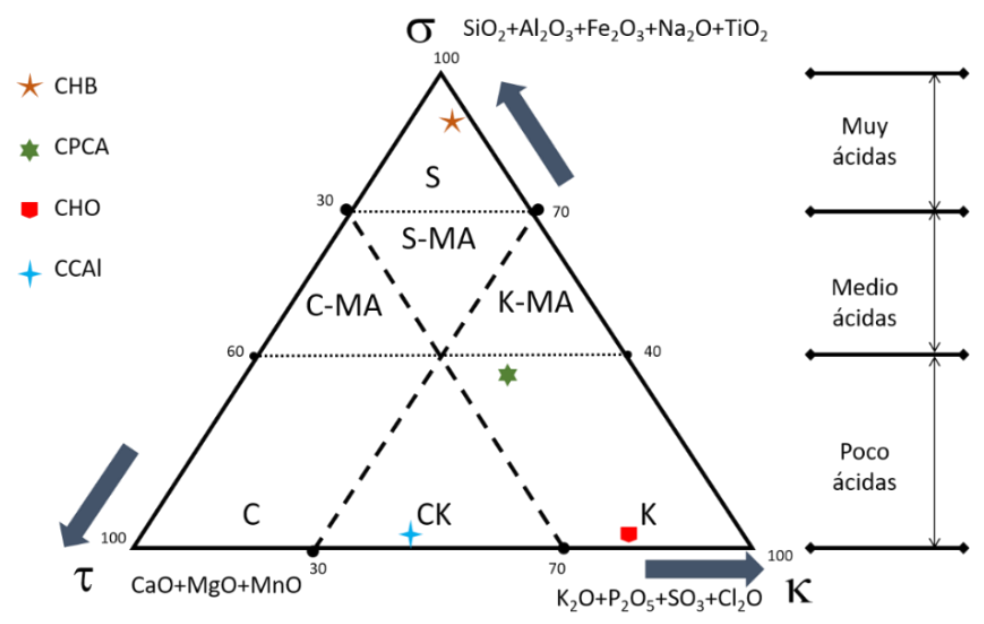

Figura 9. Diagrama ternario de composición química de cenizas de biomasa (adaptado de Vassilev et al, 2012). Representación de las cenizas estudiadas: ceniza de hoja de bambú (CHB), ceniza de paja de caña de azúcar (CPCA), ceniza de hueso de oliva (CHO) y ceniza de cáscara de almendra (CCAl).

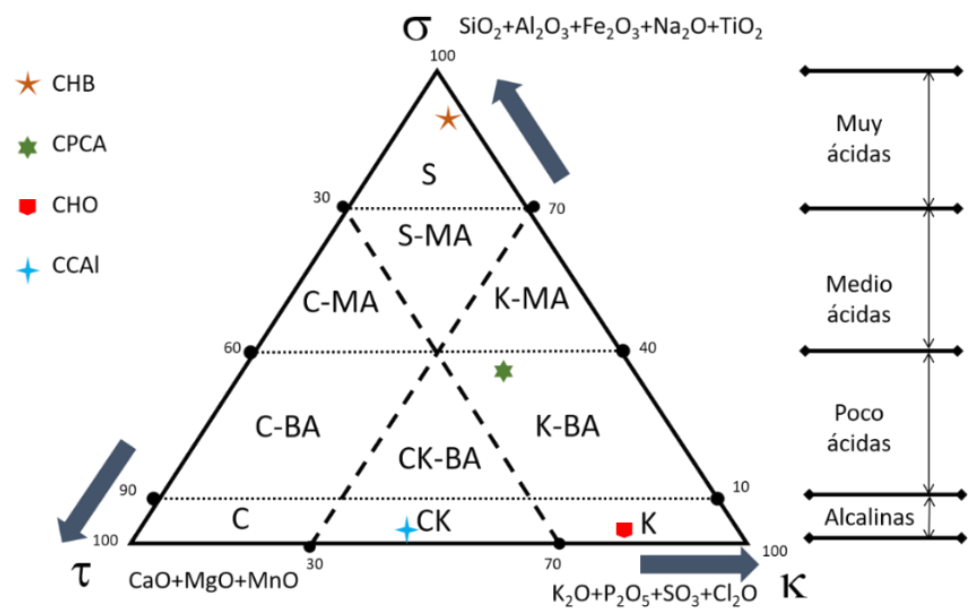

Figura 10. Nueva propuesta de diagrama ternario para la distribución de las áreas de composición de las cenizas de biomasa. 
De acuerdo con la nueva distribución en el diagrama ternario, se puede considerar que los materiales puzolánicos se encontrarán en las zonas S, S-MA, K-MA y K-BA (de mayor a menor reactividad puzolánica (siempre y cuando se trate de material amorfo y el contenido de sílice cristalina sea bajo). Por otra parte, consideraremos cenizas aptas para la activación alcalina, aquellas que están en las zonas $\mathrm{C}, \mathrm{CK}$ y K.

\subsection{Evaluación de la huella de carbono de nuevos conglomerantes con cenizas de biomasa vegetal} Partiendo de la consideración de que las cenizas, de por sí, no llevan asociada ninguna emisión de $\mathrm{CO}_{2}$, puesto que son neutras respecto a este indicador medioambiental, es razonable considerar que, en principio, los nuevos conglomerantes preparados tendrán una menor huella de carbono que los convencionales.

En el caso de los conglomerantes basados en el cemento Portland (OPC), debe considerarse que para la fabricación de 1 tonelada de dicho cemento Portland, se estima una emisión de 1 tonelada de $\mathrm{CO}_{2}$ (Phair, 2006). Es por ello que la sustitución parcial de cemento Portland por una adición suplementaria supondrá una reducción en la huella de carbono, siempre y cuando el procesado de la adición no conlleve consumos importantes de energía. En el caso de las cenizas de biomasa, es siempre conveniente su molienda (Payá et al., 2010). La molienda de estas cenizas suele ser sencilla, ya que se trata de materiales blandos. En la Tabla 1, se muestran los valores de las dosificaciones empleadas para el cálculo, mientras que en la Tabla 2 se muestran los valores de $\mathrm{CO}_{2}$ asociados al cemento Portland y a la molienda de las cenizas CHB y CPCA. Para el cálculo, se supone una molienda en laboratorio (molino de bolas de 1 litro de capacidad, potencia $0.3 \mathrm{~kW}$ ) durante un tiempo (min) y una cantidad de material por molienda $(\mathrm{g})$. Se considera que la equivalencia entre energía eléctrica y emisiones de $\mathrm{CO}_{2}$ es de $0.25 \mathrm{kgCO}_{2} / \mathrm{kWh}$ (IDAE, 2018).

En el caso de los cementos de activación alcalina, debe tenerse en cuenta el proceso de molienda de la escoria (ESC) y de las cenizas CHO y CCAl (ver Tabla 1) así como las cantidades de reactivos químicos $(\mathrm{NaOH}$ ó $\mathrm{KOH})$. Para el cálculo del $\mathrm{CO}_{2}$ emitido, se debe considerar el $\mathrm{CO}_{2}$ correspondiente a los procesos de molienda y el $\mathrm{CO}_{2}$ asociado a los activadores. En el caso de los reactivos químicos, supondremos $1.12 \mathrm{kgCO}_{2} / \mathrm{kg} \mathrm{NaOH}$ (Mellado, 2014) y $1.934 \mathrm{kgCO}_{2} / \mathrm{kgKOH}$ (Biograce, 2011).

Tabla 1. Dosificaciones de los diferentes sistemas estudiados y condiciones de molienda. OPC: cemento Portland; ESC: escoria de alto horno; CHB: ceniza de hoja de bambú; CPCA: ceniza de paja de caña de azúcar; $\mathrm{CHO}$ : ceniza de hueso de oliva; CCAl: ceniza de cáscara de almendra.

\begin{tabular}{|c|c|c|c|c|c|c|}
\hline \multirow{2}{*}{ Sistema } & \multicolumn{4}{|c|}{ Componentes (kg) de la mezcla } & \multicolumn{2}{c|}{$\begin{array}{c}\text { Condiciones molienda } \\
\text { (g-min) }\end{array}$} \\
\cline { 2 - 7 } & OPC & ESC & Ceniza & Reactivo & ESC & Ceniza \\
\hline OPC & 1 & 0 & 0 & 0 & - & - \\
\hline OPC/CHB & 0.75 & 0 & 0.25 & 0 & - & $300-40$ \\
\hline OPC/CPCA & 0.80 & 0 & 0.20 & 0 & - & $300-40$ \\
\hline ESC/NaOH & 0 & 1 & 0 & 0.19 & $450-30$ & - \\
\hline ESC/KOH & 0 & 1 & 0 & 0.18 & $450-30$ & - \\
\hline ESC/CHO & 0 & 1 & 0.25 & 0 & $450-30$ & $250-10$ \\
\hline ESC/CCAl & 0 & 1 & 0.20 & 0 & $450-30$ & $250-10$ \\
\hline
\end{tabular}

En la Tabla 2 se pueden comparar las emisiones de $\mathrm{CO}_{2}$ asociadas a cada conglomerante. Podemos destacar en primer lugar que la sustitución parcial de OPC por cenizas supone una reducción moderada en las emisiones, ligeramente inferior al porcentaje de sustitución realizado. 
Sin embargo, en la activación alcalina de escoria por vía reactivo químico $(\mathrm{KOH}, \mathrm{NaOH})$ se observa una disminución muy considerable, del orden del 60-70\% dependiendo del tipo de hidróxido. La sustitución de los reactivos químicos por residuos procedentes de la combustión de algunas biomasas, como el hueso de oliva o la cáscara de almendra, supone un ahorro medioambiental importante: se trata de reducciones superiores al $90 \%$ con respecto al cemento Portland, y entre 65 y $80 \%$ con respecto a los cementos de escoria activada con hidróxido alcalino. Por una parte, se disminuye el consumo de reactivos de síntesis, y, por otra, se da salida a un residuo que podría tener una difícil gestión. De esta manera, se puede potenciar la economía circular, puesto que un residuo de la actividad energética se valoriza en la elaboración de materiales cementantes, con un procesado escaso y con unos resultados magníficos a nivel de prestaciones mecánicas.

Tabla 2. Emisiones de $\mathrm{CO}_{2}$ (en $\mathrm{kg}$ de $\mathrm{CO}_{2}$ por $\mathrm{kg}$ de binder, siendo el binder: OPC, OPC+ceniza ó

ESC) asociadas a cada uno de los diferentes sistemas estudiados (OPC: cemento Portland; ESC: escoria de alto horno; CHB: ceniza de hoja de bambú; CPCA: ceniza de paja de caña de azúcar; CHO: ceniza de hueso de oliva; $\mathrm{CCAl}$ : ceniza de cáscara de almendra).

\begin{tabular}{|c|c|c|c|c|c|}
\hline \multirow{2}{*}{ Sistema } & \multicolumn{2}{|c|}{ Emisiones de CO2 $\mathbf{~ k g ) ~ p o r ~ k g ~ d e ~ b i n d e r ~}$} & \multirow{2}{*|}{$\begin{array}{c}\text { Emisión total } \\
\text { (kgCO//kg binder) }\end{array}$} \\
\cline { 2 - 5 } & OPC & ESC & Ceniza & Reactivo & 1.000 \\
\hline OPC & 1.000 & 0.000 & 0.000 & 0.000 & 0.786 \\
\hline $\mathrm{OPC} / \mathrm{CHB}$ & 0.750 & 0.000 & 0.036 & 0.000 & 0.829 \\
\hline $\mathrm{OPC} / \mathrm{CPCA}$ & 0.800 & 0.000 & 0.029 & 0.000 & 0.298 \\
\hline $\mathrm{ESC} / \mathrm{NaOH}$ & 0.000 & 0.083 & 0.000 & 0.215 & 0.430 \\
\hline $\mathrm{ESC} / \mathrm{KOH}$ & 0.000 & 0.083 & 0.000 & 0.347 & 0.096 \\
\hline $\mathrm{ESC} / \mathrm{CHO}$ & 0.000 & 0.083 & 0.013 & 0.000 & 0.093 \\
\hline $\mathrm{ESC} / \mathrm{CCAl}$ & 0.000 & 0.083 & 0.010 & 0.000 & \\
\hline
\end{tabular}

\subsection{Consideraciones sobre la durabilidad.}

Desde el punto de vista de la reactividad, en el caso de uso de cenizas de biomasa vegetal se consideran importantes tanto la componente ácida $\left(\mathrm{SiO}_{2}\right)$ como la alcalina $\left(\mathrm{K}_{2} \mathrm{O}\right.$ y $\left.\mathrm{CaO}\right)$. Sin embargo, el análisis de la durabilidad de los materiales elaborados debe tenerse en cuenta la presencia de otros componentes. En el caso de la CHB, situado en la zona S (ver Figura 8) vemos que el contenido de cloruro es muy bajo $(0.1 \%)$ y, probablemente, tendría nula incidencia relacionada con la corrosión de armaduras. Sin embargo, para cenizas en la zona K-BA, como la CPCA, se deben considerar los elevados contenidos en cloruro (4.3\%) así como el contenido en $\mathrm{SO}_{3}(6.3 \%)$ que podría activar procesos de expansión por formación de etringita.

En el caso de las cenizas aplicadas para la activación alcalina de la escoria, se puede destacar que, en ambos casos, $\mathrm{CHO}$ y CCAl, los contenidos en cloruro son muy bajos ( $0.32 \%$ y $0.29 \%$ respectivamente), lo cual no tendrá una incidencia significativa en la corrosión de armaduras.

\section{CONCLUSIONES}

Las cenizas de biomasa constituyen buenas materias primas para la elaboración de materiales cementantes. El origen de la biomasa condiciona claramente la naturaleza química de las cenizas, y consecuentemente las posibles aplicaciones en cementos. La ceniza de hoja de bambú se considera una excelente puzolana dado su elevado contenido en sílice, comparable al humo de sílice y a la ceniza de cáscara de arroz. 
La ceniza de paja de caña de azúcar contiene menor cantidad de sílice y una elevada proporción de cloruros. Ambas cenizas pueden ser usadas como materiales de sustitución de cemento Portland, si bien el factor cloruro debe ser tenido en lo que respecta a la corrosión de armaduras. Por otra parte, tanto la ceniza de hueso de oliva como la ceniza de cáscara de almendra son consideradas alcalinas, por sus elevados contenidos en potasio y calcio. Estos componentes pueden ser usados como activadores en cementos de activación alcalina. Su composición química, muy diferente de la encontrada para la ceniza de paja de caña de azúcar, hace que su clasificación deba ser diferente, por lo que se ha propuesto una modificación al diagrama ternario propuesto por Vassilev et al (2012), añadiendo unas nuevas zonas. Finalmente, se ha comparado la contribución a las emisiones de $\mathrm{CO}_{2}$ de los posibles cementos que contienen las cenizas estudiadas, concluyendo que los beneficios en términos de huella de carbono son significativos si se emplean cenizas de biomasa. Se ha demostrado que existe una reducción en la emisión de $\mathrm{CO}_{2}$, en especial en los cementos de activación alcalina, donde se ha reducido la huella de carbono entre 65 y $80 \%$ con respecto a los cementos activados con hidróxido alcalino (de sodio o potasio).

\section{AGRADECIMIENTOS}

A las empresas Cementval, Aceites Sahúco y Borges por el suministro de muestras. Al servicio de microscopia electrónica de la Universitat Politècnica de València.

\section{REFERENCIAS}

Andrew, R.M. (2018), Global $\mathrm{CO}_{2}$ emissions from cement production. Earth System Science Data 10:195-217. https://doi.org/10.5194/essd-10-195-2018

BioGrace GHG calculation tool, List of Additional Standard Values, version 1 (2011). Intelligent Energy Europe Programme.

IDAE 2011. S. Ministerio de Industria, Energía y Turismo, Secretaría de Estado de Energía, Madrid, http://www.idae.es/, (accessed 12 March 2018).

Jankovsky, O., Pavlokova, M., Sedmidubsky, D., Bousa, D., Lojka, M., Pokorny, J., Zaleska, M., Pavlik, Z. (2017). Study on pozzolana activity of wheat straw ash as potential admixture for blended cements. Ceramics-Silikáty 61(4):327-339. https://doi.org/10.13168/cs.2017.0032

James, A.K., Thring, R.W., Helle, S., Ghuman, H.S. (2012), Ash Management Review-Applications of Biomass Bottom Ash. Energies, 5:3856-3873. https://doi.org/doi:10.3390/en5103856

Johnson, E. (2009), Goodbye to carbon neutral: Getting biomass footprints right. Environmental Impact Assessment Review, 29(3):165-168. https://doi.org/10.1016/j.eiar.2008.11.002

Jenkins, B.M., Baxter, L.L., Miles Jr., T.R., Miles, T.R. (1998), Combustion properties of biomass. Fuel Processing Technology. 54:17-46. https://doi.org/10.1016/S0378-3820(97)00059-3

Mellado, A., Catalán, C., Bouzón, N., Borrachero, M. V., Monzó, J. M., Payá, J. (2014), Carbon footprint of geopolymeric mortar: Study of the contribution of the alkaline activating solution and assessment of an alternative route. RSC Advances, 4(45):23846-23852. https://doi.org/10.1039/c4ra03375b

Moraes, M.J.B., Moraes, J.C.B., Tashima, M.M., Akasaki, J.L., Soriano, L., Borrachero, M.V., Payá, J. (2019), Production of bamboo leaf ash by auto-combustion for pozzolanic and sustainable use in cementitious matrices. Construction and Building Materials, 208:369-380. https://doi.org/10.1016/j.conbuildmat.2019.03.007

Moraes, J.C.B., Queiroz, D.C.A., Akasaki, J.L., Melges, J.L.P., Borrachero, M.V., Payá, J., Tashima, M.M. (2016), Possibilities of reusing sugar cane straw ash in the production of alternative binders. Key Engineering Materials, 668:304-311. https://doi.org/10.4028/www.scientific.net/KEM.668.304 
Payá, J., Monzó, J., Borrachero. M.V. (2010), Outstanding aspects on the use of rice husk ash and similar agrowastes in the preparation of binders. in I Pro-Africa conference: Non conventional building materials based on agroindustrial wastes (Pirassununga, São Paulo, Brasil, 18-19 octubre, ISSN 21776334).

John W. Phair, J.W. (2006), Green chemistry for sustainable cement production and use. Green Chemistry, 8:763-780. https://doi.org/10.1039/B603997A

Pinheiro, S.M.M., Font, A., Soriano, L., Tashima, M.M., Monzó, J., Borrachero, M.V., Payá, J. (2018), Olive-stone biomass ash (OBA): an alternative alkaline source for the blast furnace slag activation. Construction and Building Materials, 178:327-338. https://doi.org/10.1016/j.conbuildmat.2018.05.157 Shen, J., Liu, X., Zhu, S., Zhang, H., Tan, J. (2011), Effects of calcination parameters on the silica phase of original and leached rice husk ash. Materials Letters, 65(8):1179-1183. https://doi.org/10.1016/j.matlet.2011.01.034

Roselló, J., Soriano, L., Santamarina, M.P., Akasaki, J.L., Melges, J.L.P., Payá, J. (2015), Microscopic characterization of silica-rich agrowastes to be used in cement binders: bamboo and sugarcane leaves. Microscopy and Microanalysis, 21:1314-1326.http://dx.doi.org/10.1017/S1431927615015019

Vassilev, S.V., Baxter, D., Andersen, L.K., Vassileva, C.G., Morgan, T.J. (2012), An overview of the organic and inorganic phase composition of biomass. Fuel, 94:1-33. https://doi.org/10.1016/j.fuel.2011.09.030

World Energy Council. https://www.worldenergy.org/data/resources/resource/biomass/ (acceso: 21/05/2019).

Xu, W., Lo, T.Y., Wang, W., Ouyang, D., Wang, P., Xing, F. (2016), Pozzolanic Reactivity of Silica Fume and Ground Rice Husk Ash as Reactive Silica in a Cementitious System: A Comparative Study. Materials, 9(3):146. https://doi.org/10.3390/ma9030146 